\title{
Variability among Isolates of Pseudomonas syringae pv. savastanoi from the Phylloplane of the Olive
}

\author{
By G. L. ERCOLAN I \\ Istituto di Microbiologia Agraria e Tecnica, Università degli Studi di Bari, Facoltà di Agraria, \\ 70126 Bari, Italy
}

(Received 25 October 1982)

\begin{abstract}
Leaves of three or four different ages were taken from olive plants quarterly in 1974-1980. One thousand and fifty isolates of Pseudomonas syringae pv. savastanoi from the phylloplane were tested for virulence to the olive and subjected to numerical phenetic analysis using 60 unit characters. The data were analysed using unweighted average linkage (UPGMA) and single linkage clustering on the simple matching $\left(S_{S M}\right)$ and pattern $\left(S_{P}\right)$ coefficients. The isolates obtained from leaves of a given age at a given time of the year shared higher percentage similarity $(S)$ values between themselves than with the others. Cluster composition was only marginally affected by different coefficients and methods of clustering. UPGMA analysis on the $S_{S M}$ coefficient recovered $92 \%$ of the isolates in 10 major clusters at $75 \% S$. Of the isolates from leaves of the same age collected at the same time of the year, $81-99 \%$ fell in the same cluster. Conversely, $91-97 \%$ of the isolates in five of the major clusters were from leaves of the same type. Of the isolates in the other major clusters, $95-98 \%$ were from two different sources but most of the isolates from leaves of one type segregated into discrete subclusters at $85-90 \% \mathrm{~S}$. Hypothetical median organisms (HMOs) were constructed to represent all the isolates obtained from the leaves of each type each year. The resulting relationships between the HMOs confirmed those described above for the individual isolates.
\end{abstract}

\section{INTRODUCTION}

Pseudomonas syringae pv. savastanoi (Dye et al., 1980; henceforth referred to as pv. savastanoi) causes an endemic disease of the olive (Olea europaea) known as 'knot' or 'tubercle'. The primary symptoms consist of woody outgrowths that are most frequently seen on the young stems and on the branches and twigs. The leaves are rarely infected but they usually harbour pv. savastanoi on their surface.

Ercolani (1970) used a semi-selective medium to show that the density of pv. savastanoi on the phylloplane of the olive at three locations in 1969 did not increase with the severity of the disease on individual plants but was invariably greater in the spring and autumn (with a mean of $7.9 \times$ $10^{4}$ and $2.3 \times 10^{4}$ bacteria $\mathrm{cm}^{-2}$, respectively) than in the winter and summer $\left(6.6 \times 10^{3}\right.$ and 5.8 $\times 10^{2}$ bacteria $\mathrm{cm}^{-2}$, respectively). Later work with a non-selective medium in 1973-1975 confirmed these results and showed that there were characteristic fluctuations in the populations of several other heterotrophic bacteria on the phylloplane (Ercolani, 1978).

Because the olive is an evergreen, the proportion of leaves of different ages on the trees changes markedly with time. In the south-east of Italy, the emergence of new leaves begins in the spring and parallels the activity of the cambium, being intense at the end of the spring and beginning of summer, slight in mid-summer and moderate in autumn (Armenise, 1950). The leaves persist on the branches for little over a year (Morettini, 1972). These circumstances prompted field work in 1973-1976 to determine whether seasonal fluctuation in the overall density of pv. savastanoi within the leaf canopy was affected by the changing distribution of the leaves among different age classes at different times of the year. This showed that (i) pv. savastanoi was invariably present on the leaves of any age at any time of the year, and (ii) the size 
Table 1. Meteorological data recorded during the month before each sampling

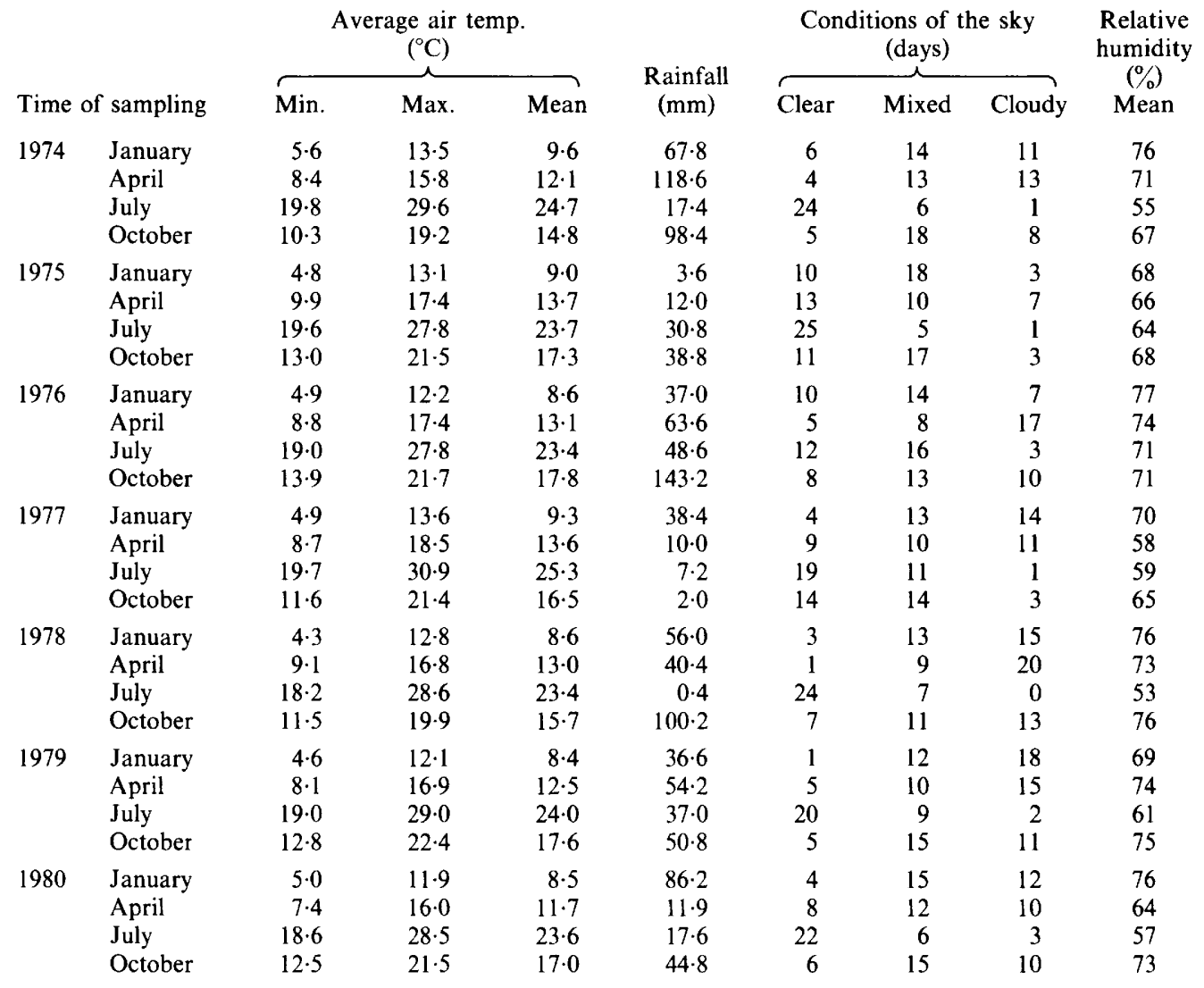

of bacterial populations on individual leaves was under the influence of first-order interactions between the age of the leaves, the time of the year when these were formed, and the time of the year when they were examined (Ercolani, 1979).

Two explanations can account for the success of such bacteria as pv. savastanoi in colonizing the surface of leaves of different ages under a wide range of environmental conditions: either the bacteria are homogeneous but highly versatile in their nutritional and physiological requirements, or they exist in nature as heterogeneous populations that alternate on the phylloplane under the combined selective pressure of the host and the environment. The work described in this paper was intended to compare the characters of several isolates of pv. savastanoi obtained from olive leaves of different ages at different times of the year.

\section{METHODS}

Sampling and isolation. The field work was done in a grove of olives (cv. Coratina) at Bitonto (Bari) in S.E. Italy. A summary of prevailing meteorological conditions is presented in Table 1.

The plant material and methods of sampling were as described before (Ercolani, 1978, 1979) with the modifications indicated below. Several unfolding leaves were mapped on the trees at the end of March, the end of June and the end of September each year in 1973-1980. Five leaf samples, each with a total surface area of 1000 $\mathrm{cm}^{2}$, were taken approximately 1, 4, 7, 10 and 13 months after mapping, starting in January, 1974, and continuing until October, 1980. Consequently, separate samples were made up of 4-, 7-and 10-month-old leaves at the end of January, 1-, 7-, 10- and 13-month-old leaves at the end of April, 1-, 4-, 10- and 13-month-old leaves at the end of July and 1-, 4-, 7- and 13-month-old leaves at the end of October each year in 1974-1980. A random sample of the 
same size as those above was also taken from unmapped leaves at each time of sampling. Thus, leaf samples from 19 different sources were obtained each year. Fifteen samples consisted of leaves of one type and four of random leaves.

Numbers of pv. savastanoi were estimated on each sample by washing the leaves, plating the wash water on nutrient agar supplemented with $50 \mathrm{mg}$ sucrose and $67 \mu \mathrm{g}$ cycloheximide $\mathrm{ml}^{-1}$, and incubating the plates $4 \mathrm{~d}$ at $26^{\circ} \mathrm{C}$. Fifty random colonies from those showing the typical morphology of pv. savastanoi and consisting of Gramnegative, oxidase-negative, arginine dihydrolase-negative, non-pectolytic, aerobic rods with polar flagella were purified, shown to be virulent for the olive, cv. Coratina, and stored on nutrient agar supplemented with $20 \mathrm{mg}$ glycerol $\mathrm{ml}^{-1}$ at $4{ }^{\circ} \mathrm{C}$ for up to 3 months.

Characterization of the isolates. Each isolate was examined for 20 phenotypic characters generated from two earlier studies (Bottalico \& Ercolani, 1970; Ercolani, 1978). The tests were those that combined a probability of error ( $p$; Sneath \& Johnson, 1972) of 0.05 or less, with a separation figure ( $C$; Gyllenberg, 1963) not significantly different from 0.5, when performed as described by Bottalico \& Ercolani (1970) and Ercolani (1978). They were colonial characteristics (transparency, production of levan and diameter $<2 \mathrm{~mm}$ ); growth in broth at $38^{\circ} \mathrm{C}$, at $\mathrm{pH} 5 \cdot 0$, at $\mathrm{pH} 7.8$ and with $3.0 \%(\mathrm{w} / \mathrm{v}) \mathrm{NaCl}$; use of malonate as a sole carbon source; acid from mannose, lactose, maltose, sucrose, meso-inositol and sorbitol; degradation of urea, tyrosine and Tween 40; resistance to oleandomycin at $15 \mu \mathrm{g} \mathrm{ml}^{-1}$ and dihydrostreptomycin at $10 \mu \mathrm{g} \mathrm{ml}^{-1}$; and pathogenicity (induction of a hypersensitive response in tobacco leaves with suspensions containing $10^{6} \mathrm{c} . \mathrm{f} . \mathrm{u} . \mathrm{ml}^{-1}$ ).

Ten isolates from each leaf sample were selected at random from those characterized as above and further examined for 60 phenotypic characters (Table 2). The characters were the 60 best separators for 128 isolates of pv. savastanoi obtained from the surface of olive leaves of different ages at different times during a pilot study in 1973. Colonial characters were examined at $4 \mathrm{~d}$ on nutrient agar containing $1 \%(\mathrm{w} / \mathrm{v})$ glucose. Nutrient broth containing $1 \%(w / v)$ glucose was inoculated with $10^{5} \mathrm{c}$.f.u. $\mathrm{ml}^{-1}$ from an exponentially growing culture and incubated for $7 \mathrm{~d}$ to observe the occurrence and the characteristics of growth under different conditions. The utilization of substances as sole carbon sources was determined according to Sands et al. (1970). The degradation of Tween 40, tyrosine and urea was tested as described by Sierra (1957), Crosse \& Garrett (1963) and Christensen (1946), respectively. The resistance to antibiotics contained in Sensi-Disks (BBL) was recorded at $24 \mathrm{~h}$ on plates of Antibiotic Medium 3 (Difco) solidified with $1.2 \%(\mathrm{w} / \mathrm{v})$ agar and seeded with $4.5 \times 10^{6}$ c.f.u. $\mathrm{ml}^{-1}$ from an exponentially growing culture. The test for induction of a hypersensitive reaction in tobacco leaves was done according to Klement (1963). The incubation temperature was $26^{\circ} \mathrm{C}$ for all tests except the hypersensitive reaction in tobacco leaves, for which it ranged between 20 and $26^{\circ} \mathrm{C}$. Duplication of three isolates throughout the study showed that observational error exceeded $5 \%$ in three tests (production of translucent colonies, formation of a pellicle in broth culture and degradation of tyrosine, for which it was $6.4,5.9$ and $7 \cdot 1 \%$, respectively) and remained below $5 \%$ in the others and averaged $3.2 \%$ over all the tests. These may be considered acceptable values (Sneath \& Johnson, 1972).

Clustering of the isolates. The results of all tests were recorded in the '0' (negative) - ' 1 ' (positive) format and analysed on an IBM 370/158 computer using programs derived by S. Eberle from Wishart's (1969) Clustan 1.

The isolates examined for 60 characters were processed separately in two groups comprising the 1050 isolates from leaves of one type and the 280 isolates from random leaves, respectively. The isolates in each group were compared using the simple matching coefficient $\left(S_{S M}\right)$ and the pattern coefficient $\left(S_{P}\right)$ with both single linkage and unweighted average linkage (UPGMA) cluster analysis (Sneath \& Sokal, 1973). The clusters and subclusters were defined at the 75 and $85 \%$ similarity $(S)$ level, respectively. The vigour coefficient (Sneath, 1968) of each isolate was also measured and used for determining the mean vigour of each cluster. Clustering by the UPGMA analysis on the $S_{S M}$ and $S_{P}$ coefficients was repeated after all the isolates from the same sample had been replaced with a hypothetical median organism (HMO; Liston et al., 1963) to represent them. This resulted in simplified $S$ matrices suitable for summarizing the relationships between the isolates obtained from different samples.

The data from the 20 tests on all the isolates were used for constructing one HMO for each sample. The 105 HMOs that represented the isolates from leaves of one type and the $28 \mathrm{HMOs}$ for the isolates from random leaves were compared and clustered separately using the $S_{S M}$ coefficient and UPGMA analysis.

\section{RESULTS}

\section{Clustering of the isolates by UPGMA analysis on the $S_{S M}$ coefficient}

All but four of the 1050 isolates from leaves of one type were recovered in 10 major and 28 minor clusters (Fig. 1). Each of the major and minor clusters contained 60-134 and 2-5 isolates, respectively. The majority $(81-99 \%)$ of the isolates from a given source were recovered in one of the major clusters while the others generated the minor clusters or merged with isolates from different sources into one of the other major clusters. Altogether, $92 \%$ of the isolates were thus 


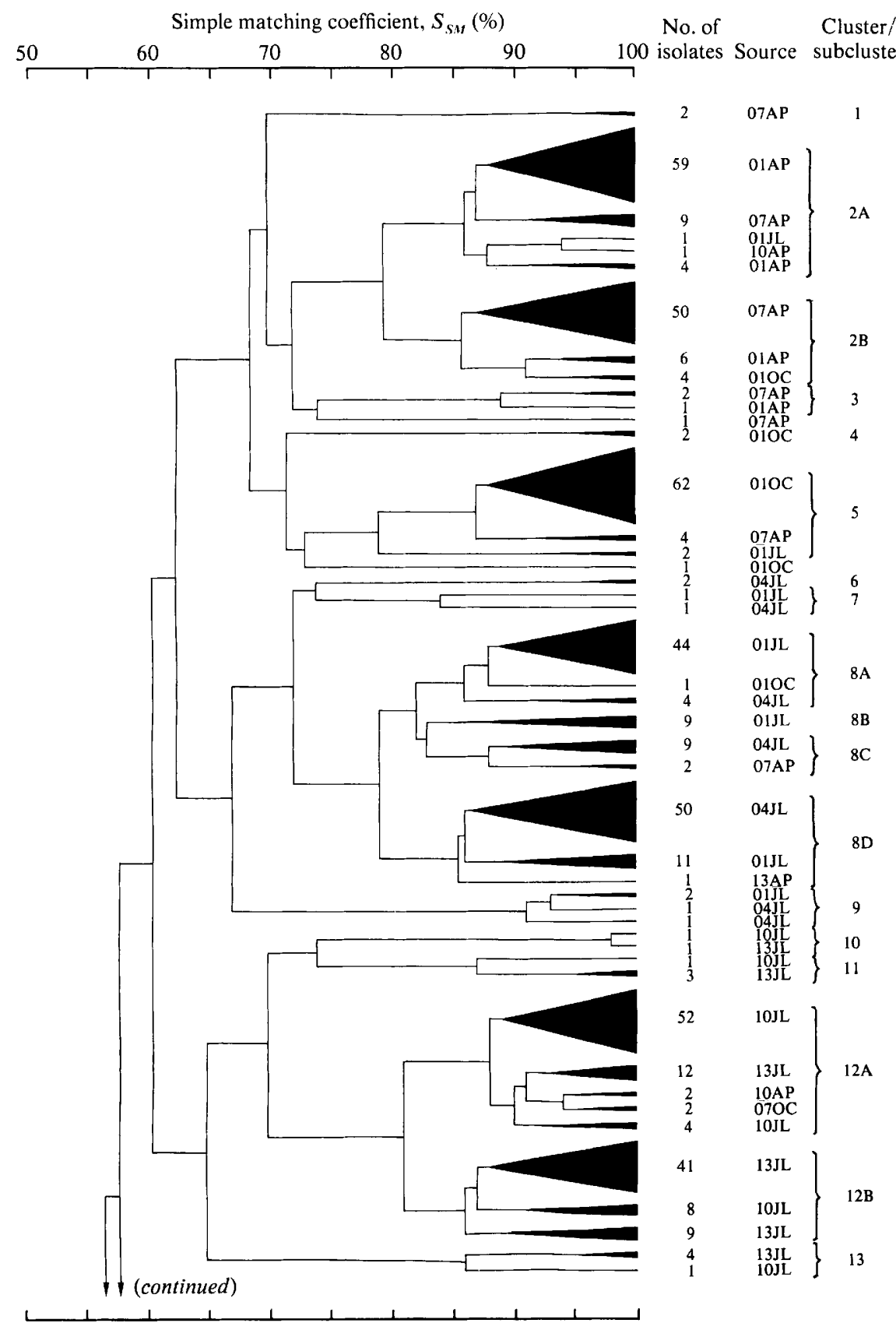

Fig. 1 (continued on opposite page). Simplified dendrogram of 1050 isolates of Pseudomonas syringae pv. savastanoi obtained from olive leaves of different ages at different times of the year in 1974-1980. The relationships shown are based on the $S_{S M}$ coefficient and unweighted average linkage clustering (UPGMA). The isolates are identified by the age of the leaves from which they were obtained (01-, 04-, $07-, 10$ - and 13-month-old) and the time of the year when the leaves were that age (JN $=$ January; AP $=$ April; $\mathrm{JL}=$ July; $\mathrm{OC}=$ October . 
No. of Cluster/

Simple matching coefficient, $S_{S M}(\%)$

isolates Source subcluster
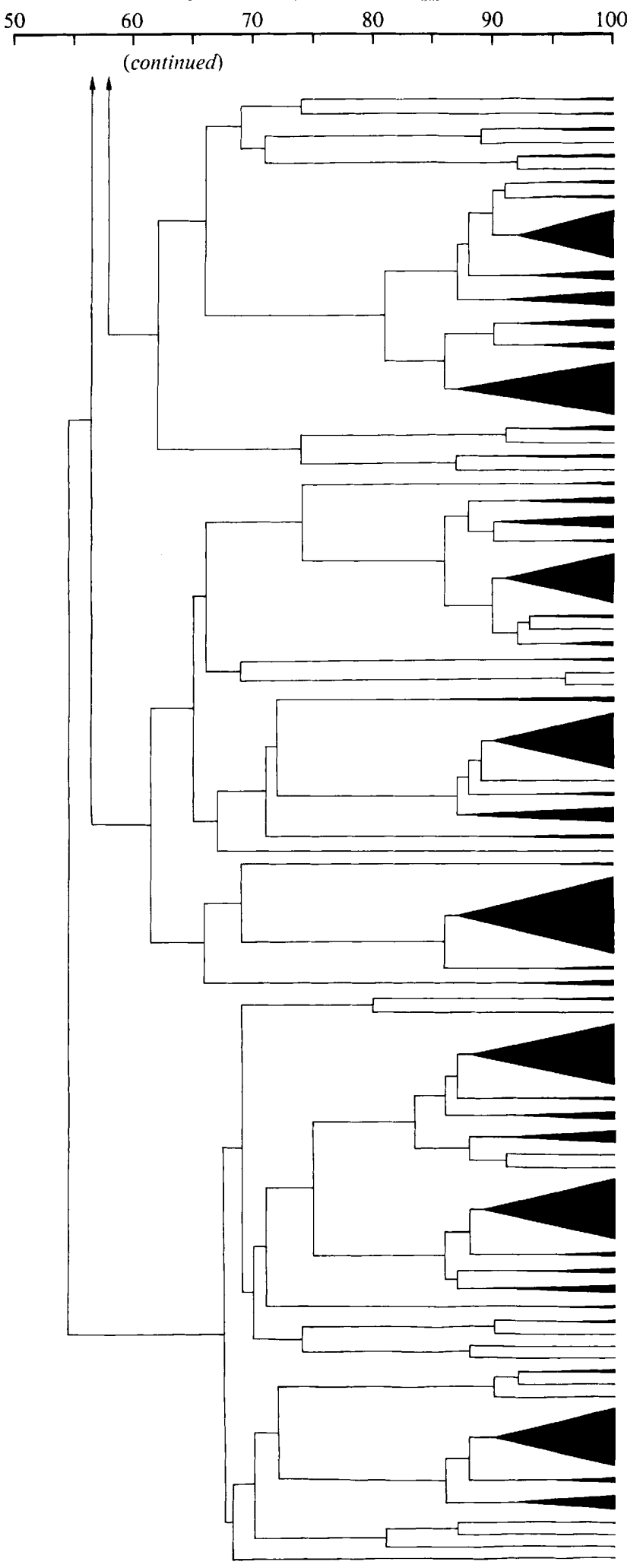

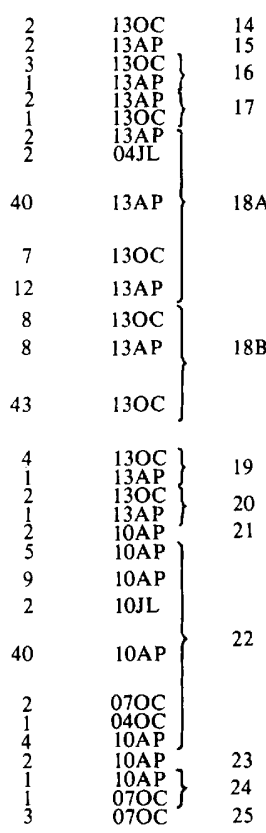

$\left.\begin{array}{ll}45 & 070 \mathrm{C} \\ 1 & \begin{array}{l}10 \mathrm{JL} \\ 2\end{array} \\ 10 \mathrm{AP}\end{array}\right\} \quad 26$

$07 \mathrm{OC}$

07OC 27

$\begin{array}{ll}07 \mathrm{OC} & 28\end{array}$

$\left.\begin{array}{lll}64 & & 04 \mathrm{OC} \\ & \\ 2 & \text { 10AP }\end{array}\right\}$

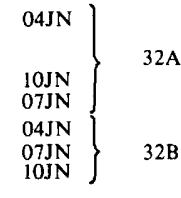

50

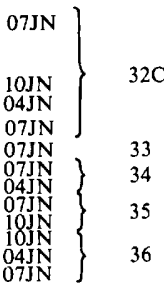

$\left.\begin{array}{l}10 \mathrm{JN} \\ 04 \mathrm{JN} \\ 10 \mathrm{JN} \\ 10 \mathrm{JN} \\ 04 \mathrm{JN} \\ 07 \mathrm{JN} \\ 07 \mathrm{JN}\end{array}\right\} 38$ 


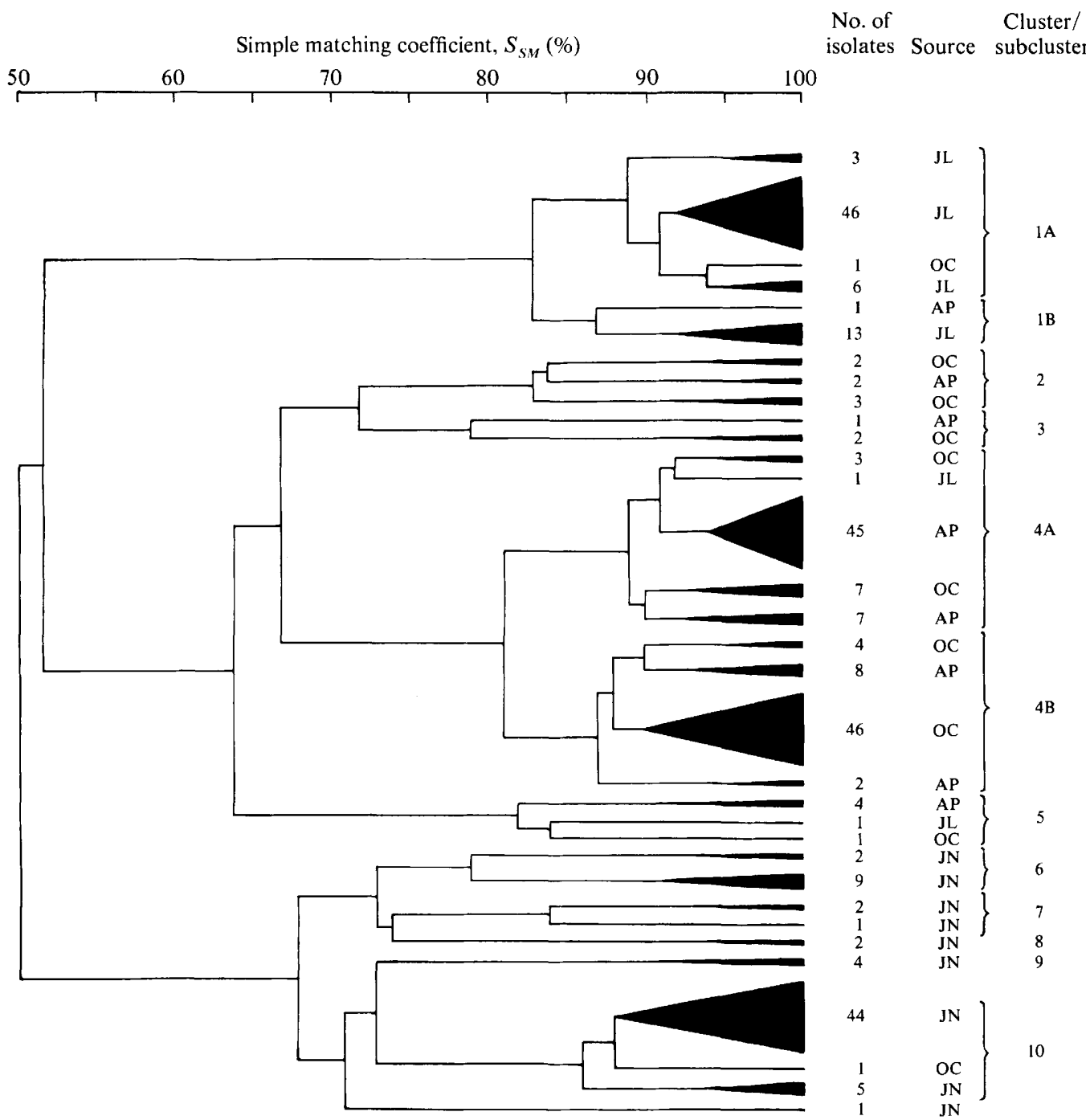

Fig. 2. Simplified dendrogram of 280 isolates of Pseudomonas syringae pv. savastanoi obtained from random samples of olive leaves in 1974-1980. The relationships shown are based on the $S_{S M}$ coefficient and unweighted average linkage clustering (UPGMA). The isolates are identified by the time of the year when they were obtained (see legend to Fig. 1).

allocated to the major clusters. Between 91 and $97 \%$ of the isolates in clusters 5, 22, 26, 29 and 37 were obtained from one source, i.e. 1-month-old leaves in October, 10-month-old leaves in April, 7-month-old leaves in October, 4-month-old leaves in October and 10-month-old leaves in January, respectively. Clusters $2,8,12,18$ and 32 accommodated $83-99 \%$ of the 70 isolates obtained from each of two sources, i.e. 1- and 7-month-old leaves in April, 1- and 4-month-old leaves in July, 10- and 13-month-old leaves in July, 13-month-old leaves in April and October, and 4- and 7-month-old leaves in January, respectively. Most of the isolates from the same source segregated into discrete subclusters but $11-31 \%$ of the isolates from each source remained intermingled with others.

Of the 280 isolates from random samples, 243 were recovered in three major and 36 in seven minor clusters (Fig. 2). Two of the major clusters contained 70 and $97 \%$ of the isolates in January 
and July, respectively; the third was divided into two subclusters, each containing 74 and $71 \%$ of the isolates obtained in April and October, respectively.

\section{Clustering of the isolates by UPGMA analysis on the $S_{P}$ coefficient}

The composition of the major clusters and their subclusters outlined in Fig. 1 remained virtually unchanged after removal of the vigour component. An exception was the isolates obtained from the younger leaves in April and July, the majority of which formed one cluster. Within this cluster, most of the isolates obtained from 1-month-old leaves in April and July formed one subcluster at $89 \% \mathrm{~S}$ and were joined by the majority of those obtained from 7 -monthold leaves in April and 4-month-old leaves in July at 79 and $76 \% \mathrm{~S}$, respectively. The resulting cluster, at one end of the $S$ matrix, was flanked by the others in a somewhat different order from that in Fig. 1. This was paralleled by the different arrangement of the HMOs in the $S_{S M} /$ UPGMA and $S_{P} /$ UPGMA matrices (see below).

Use of the $S_{P}$ instead of the $S_{S M}$ coefficient had no visible effect on the composition or relative arrangement of the clusters of isolates from random leaves, except for the occasional shift of a few isolates from one cluster to an adjacent one.

\section{Clustering of the isolates by single linkage analysis}

The clustering technique did not appreciably modify the composition or the relative arrangement of the major clusters of isolates from random leaves.

With the isolates from leaves of one type, clustering by single linkage had different effects on the analyses based on the $S_{S M}$ and $S_{P}$ coefficients. When the former was used, most of the minor clusters defined by the UPGMA analysis were absorbed into one of the 10 major clusters, but these remained separated. Merging of the minor into the major clusters also occurred with the $S_{P}$ coefficient. In addition, only three of the major clusters remained distinct. These were the clusters containing the majority of the isolates obtained from 4-month-old leaves in October, 10month-old leaves in January and other leaves in January. Of the remaining clusters formed in the UPGMA analysis, those corresponding to the isolates from 7-month-old leaves in October and to the isolates from 10- and 13-month-old leaves in July were combined at $77 \% \mathrm{~S}$, while all the others were grouped at $76 \% S$ into one aggregate corresponding to 554 individual isolates. When several UPGMA clusters merged together in the single linkage analysis, the corresponding isolates formed discrete subclusters. Use of single linkage analysis left the composition of the UPGMA subclusters almost unchanged.

\section{Clustering of the HMOs}

The results with the HMOs generated from data on 60 characters differed little from those obtained when 20 characters were used. The comments below apply to both sets of HMOs.

All the HMOs representing the isolates from leaves of one type were recovered in 10 clusters by $S_{S M}$ /UPGMA analysis (Fig. 3). The HMOs representing the isolates from leaves of the same type always fell in the same cluster. Five clusters were each composed of seven HMOs representing the isolates from leaves of the same type. Each of the other five clusters contained 14 HMOs that represented the isolates from leaves of two types, but the HMOs corresponding to the isolates from leaves of one type segregated into discrete subclusters. The resulting dendrogram reproduced in simplified form the relationships already observed between the majority of individual isolates, without intermediates between the clusters (Figs 1 and 3). $S_{P} /$ UPGMA analysis recovered the HMOs in nine clusters (Fig. 3). One large cluster contained the HMOs representing the isolates obtained from leaves of four types (1- and 7-month-old in April and 1- and 4-month-old in July) but the HMOs corresponding to the isolates from each source remained distinct at subcluster level. The other eight clusters maintained the same composition resulting from $S_{S M} /$ UPGMA analysis but were arranged in different order.

The HMOs representing the isolates from random leaves fell in three clusters of identical composition by both $S_{S M}$ and $S_{P} /$ UPGMA analysis. The two peripheral clusters contained the HMOs for the isolates of January and July, respectively. The central cluster accommodated two subclusters corresponding to the HMOs for the isolates of April and October, as noted before with the individual isolates. 


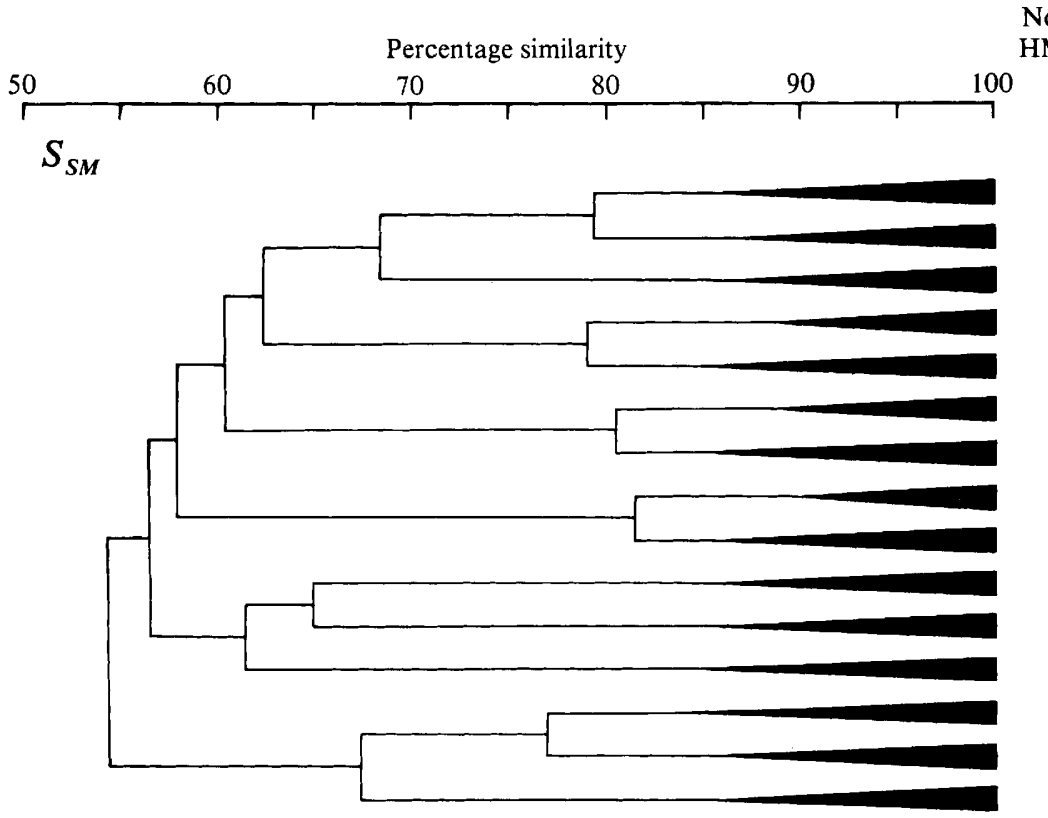

No. of

Cluster/

HMOs Source subcluster

\section{7}

01AP $\quad 1 \mathrm{~A}$

07AP $1 \mathrm{~B}$

0IOC 2

$01 \mathrm{JL} \quad 3 \mathrm{~A}$

04JL 3B

$10 \mathrm{JL} \quad 4 \mathrm{~A}$

$13 \mathrm{JL} \quad 4 \mathrm{~B}$

13AP $5 \mathrm{~A}$

$130 \mathrm{C} 5 \mathrm{~B}$

10AP 6

07OC 7

04OC 8

04JN 9A

07JN 9B

10JN 10

$S_{P}$

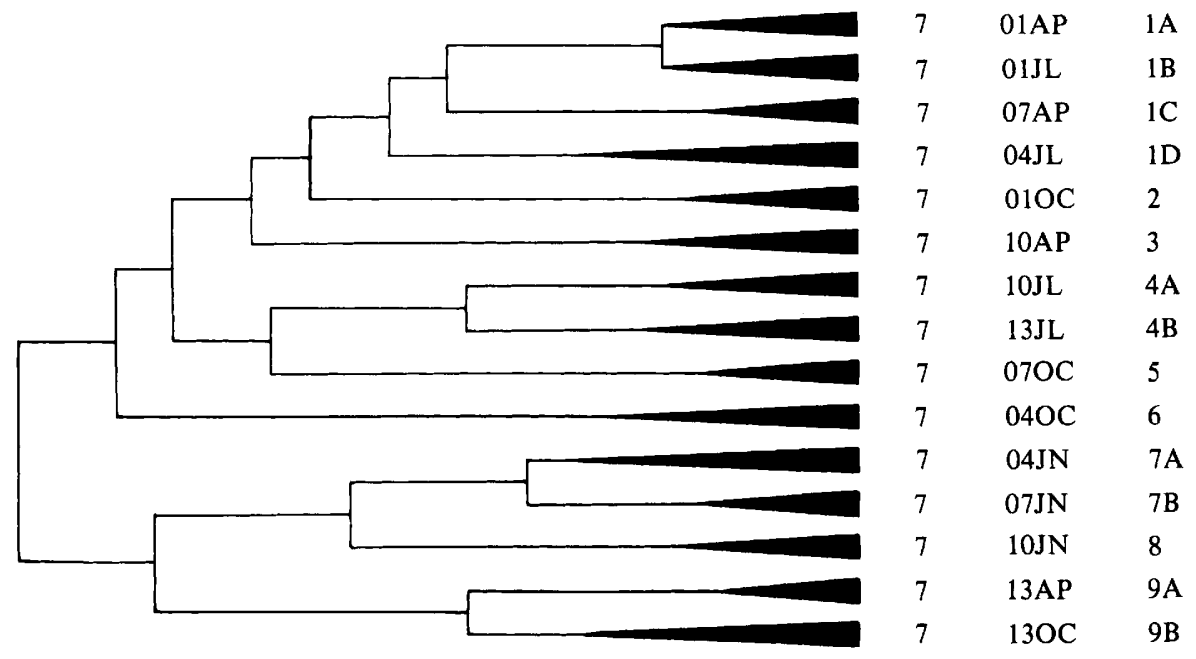

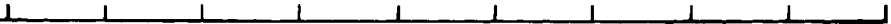

Fig. 3. Simplified dendrograms of the hypothetical median organisms (HMOs) representing the isolates of Pseudomonas syringae pv. savastanoi obtained from olive leaves of different ages at different times of the year in 1974-1980. The relationships shown are based on unweighted average linkage clustering (UPGMA) with the $S_{S M}$ coefficient (above) and the $S_{P}$ coefficient (below). The HMOs are identified by the age of the leaves from which the corresponding isolates were obtained and the time of the year when the leaves were that age (see legend to Fig. 1). 
The similarity between the HMOs was not obviously correlated with the calendar year in which the corresponding isolates were obtained. Each cluster or subcluster defined at $85 \% \mathrm{~S}$ contained one to three HMOs with a higher mean resemblance to all other HMOs in the same group (Fig. 4). Such HMOs, however, were not generated with significantly higher frequency during any particular year.

\section{Characterization of the major clusters}

Table 2 shows the distribution of test responses to the 10 major clusters/subclusters illustrated in Fig. 1. These were the groups formed by $S_{S M} /$ UPGMA analysis with the isolates from leaves of one type. No single test was cluster specific or suitable for discriminating between all the clusters. All the tests, however, proved useful for characterizing at least some of the clusters. The characters reported in Table 2 for clusters/subclusters 12B, 18A, 18B and 37 applied also to the four major groups of isolates from random leaves, i.e., 1A, 4A, 4B and 10, respectively (Fig. 2). Each of these groups contained the majority of the isolates obtained from random leaves in July, April, October and January.

The mean vigour of the clusters/subclusters in Table 2 ranged from 0.513 to 0.658 but was not significantly correlated with the age of the leaves from which the isolates were obtained or with the time of the year when the leaves were that age.

\section{DISCUSSION}

The characterization of microbial populations in natural habitats has been attempted by several authors with different methods, including numerical taxonomy (Oliver \& Colwell, 1974; Goodfellow et al., 1976), numerical profiles (Griffiths \& Lovitt, 1980), principal component and factor analysis (Rosswall \& Kvillner, 1978), reciprocal averaging (Houba \& Remacle, 1980), time-series analysis (Fry et al., 1981) and estimates of diversity by Shannon information index or Simberloff rarefaction method (Martin \& Bianchi, 1980; Mills \& Wassel, 1980). In this study, numerical phenetic analysis of epiphytic isolates of pv. savastanoi by single linkage or average linkage on the $S_{S M}$ or $S_{P}$ coefficients proved adequate to demonstrate a correlation between the source of the isolates and their phenotypic characters.

The populations of pv. savastanoi found on different samples of leaves of the same type over the years 1974-1980 shared a greater number of cultural and physiological characters with one another than with populations colonizing the leaves of a different type over the same years. The use of different similarity coefficients and clustering procedures did not affect the general validity of this conclusion but marginally modified the results of the analyses when the relationships between the isolates were defined at the $85 \% S$-level. The well known chaining effect of single linkage clustering (Sneath \& Sokal, 1973) became evident when the threshold for cluster formation was set at $75 \% S$, especially if the $S_{P}$ coefficient was used.

The correlation between source and phenotypic characters was confirmed with the isolates obtained from random leaves at three of the four sampling times each year. As a general rule the distribution of characters to the collections of isolates from random leaves could be anticipated from currently available data, i.e. the seasonal fluctuation in the proportion of leaves of different ages within the canopy of the olive (Morettini, 1972), the average density of pv. savastanoi on the leaves of different ages at different times of the year (Ercolani, 1979) and the composition of bacterial populations on the leaves of any one type (Fig. 1). On the assumption that the random samples taken during this work were representative of the true distribution of leaves, 74, 70 and $67 \%$ of the isolates in April, October and January could be expected to have the characters given in Table 2 for clusters/subclusters $18 \mathrm{~A}, 18 \mathrm{~B}$ and 37 , respectively. The actual values were 74,71 and $70 \%$. In July, however, approximately 20,26 and $54 \%$ of the isolates were expected with the characters of subclusters $8 \mathrm{D}, 12 \mathrm{~A}$ and $12 \mathrm{~B}$ (Table 2) but the actual values were $<1,19$ and $74 \%$, respectively. This finding can probably be explained by recurrent sampling errors in July, due perhaps to the spatial distribution of leaves of different ages at that time of the year. Additional data from 'random' samples in July should clarify this point. 


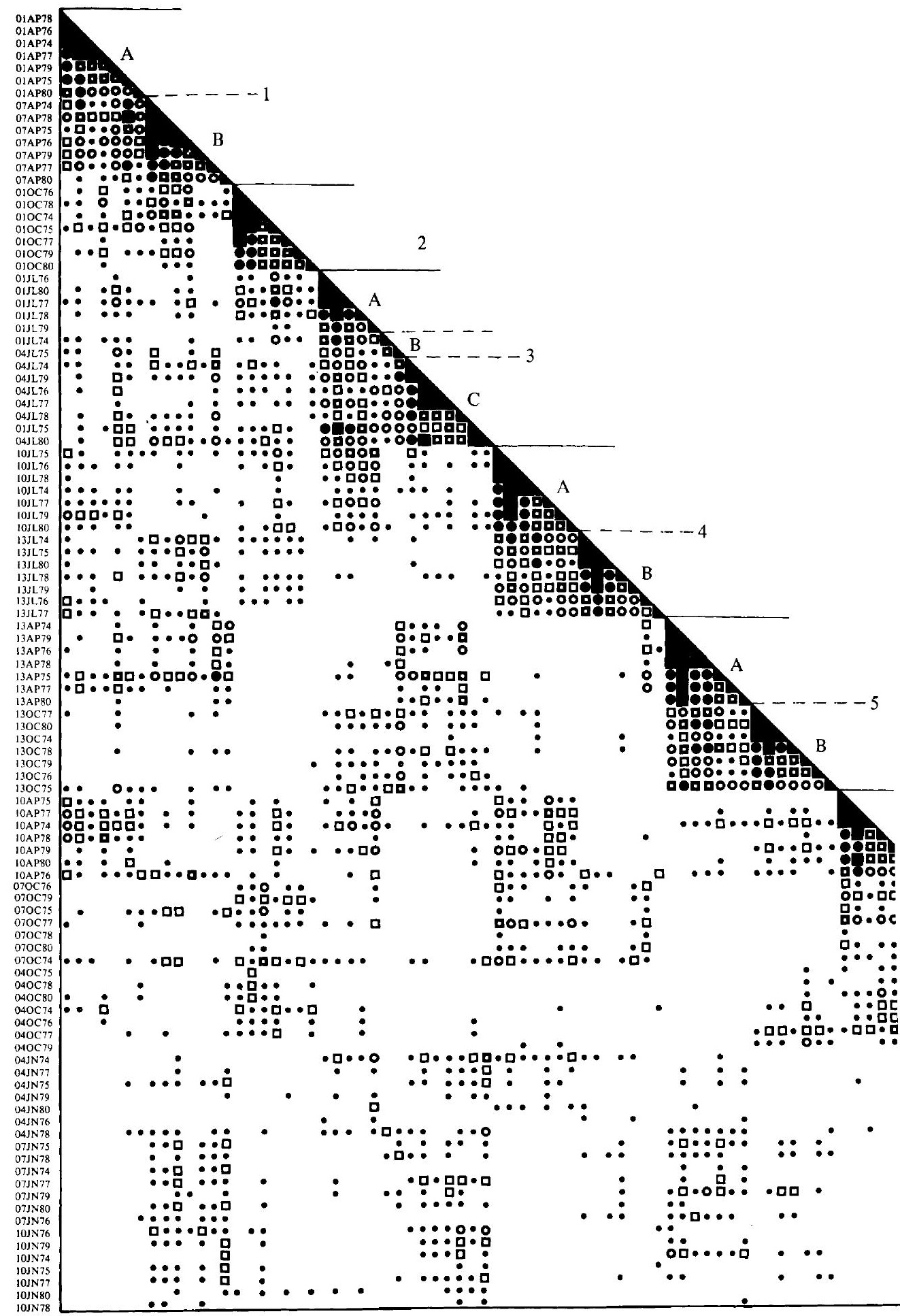


Fig. 4. Sorted similarity matrix of the hypothetical median organisms (HMOs) representing the isolates of Pseudomonas syringae pv. savastanoi obtained from olive leaves of different ages at different times of the year in 1974-1980. The relationships shown are based on the $S_{S M}$ coefficient and unweighted average linkage clustering (UPGMA). The HMOs are identified by the age of the leaves from which the corresponding isolates were obtained and the month and year when the isolations were made (see legend to Fig. $1 ; 74-80=1974-1980$ ).

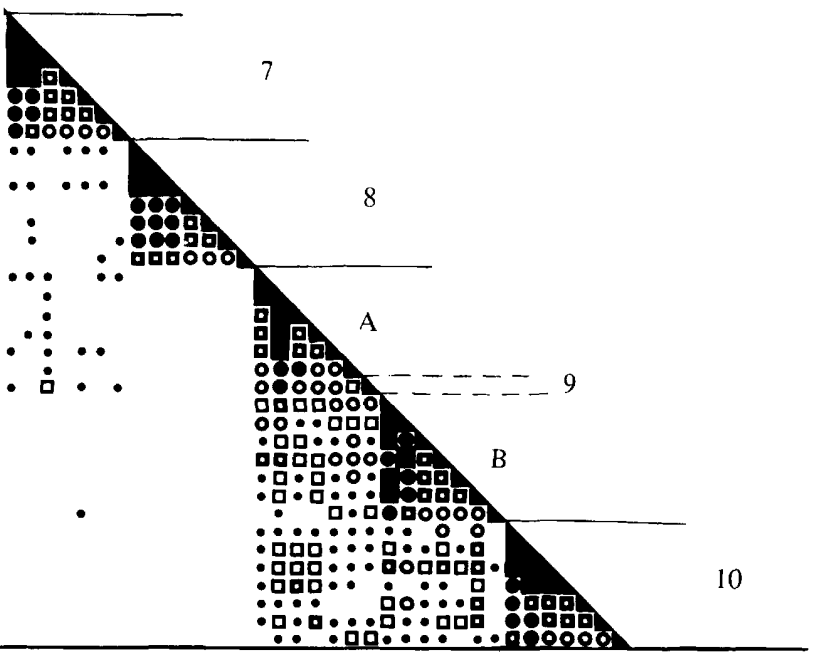




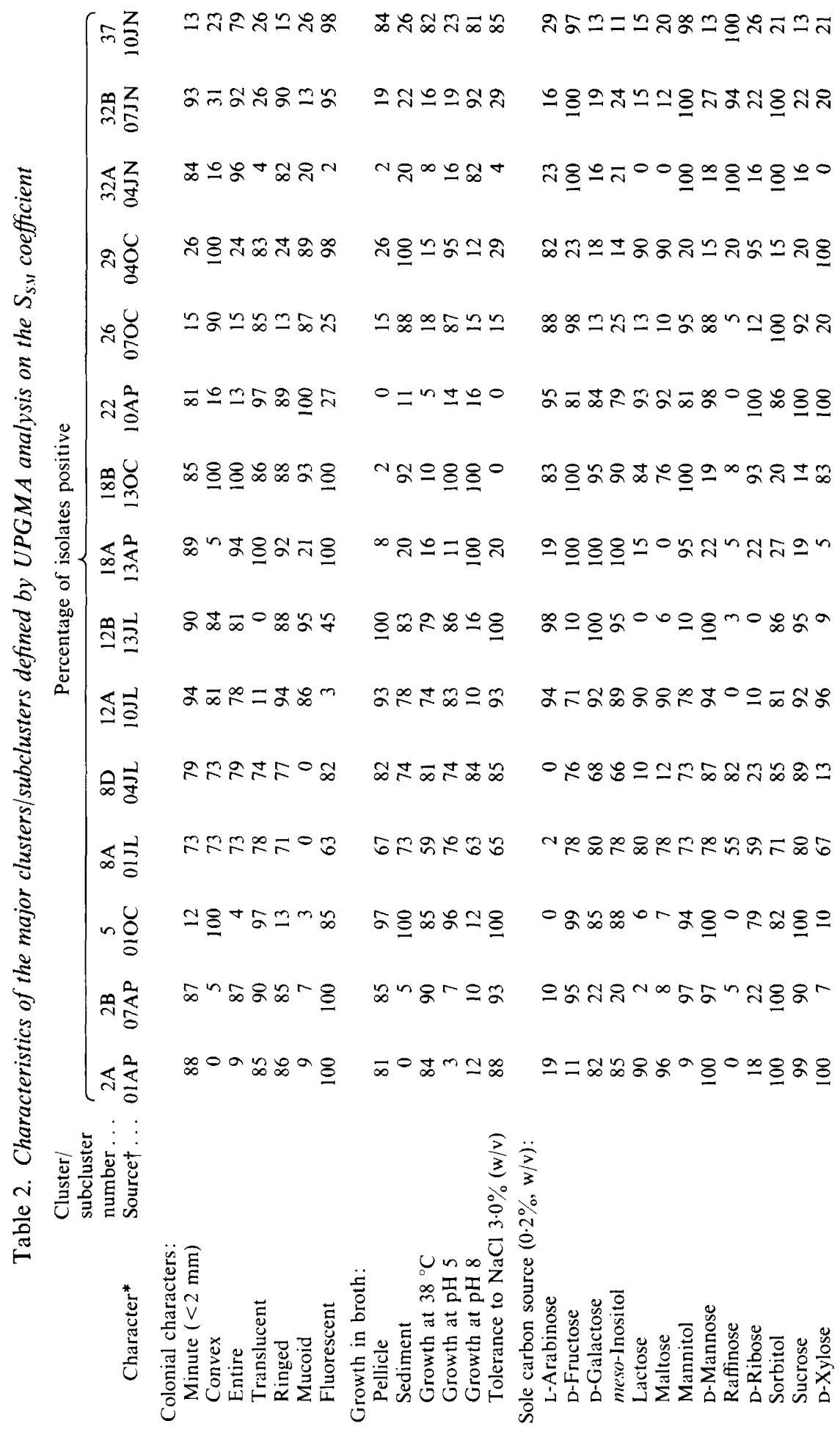




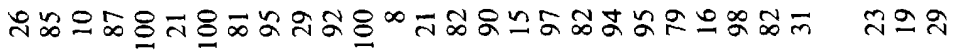

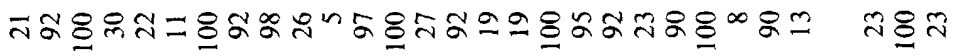

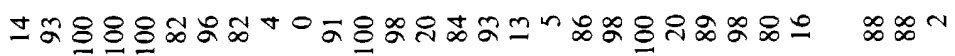

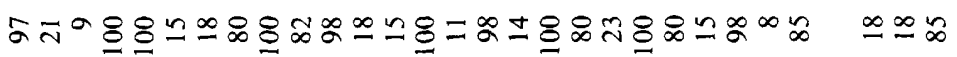

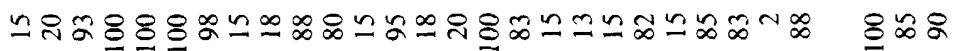

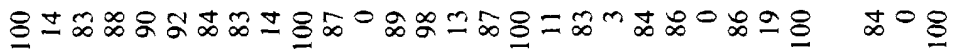

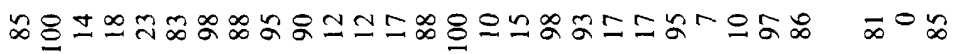

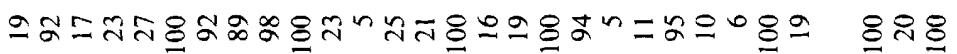
○レ

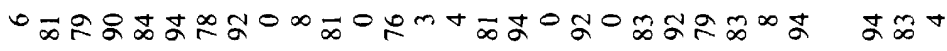

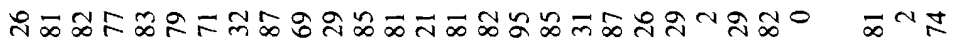

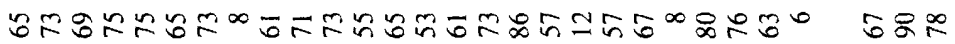

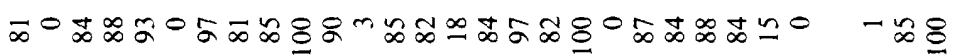

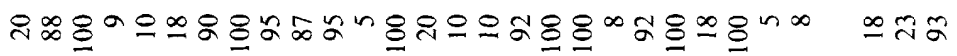

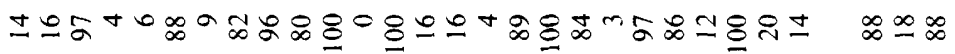

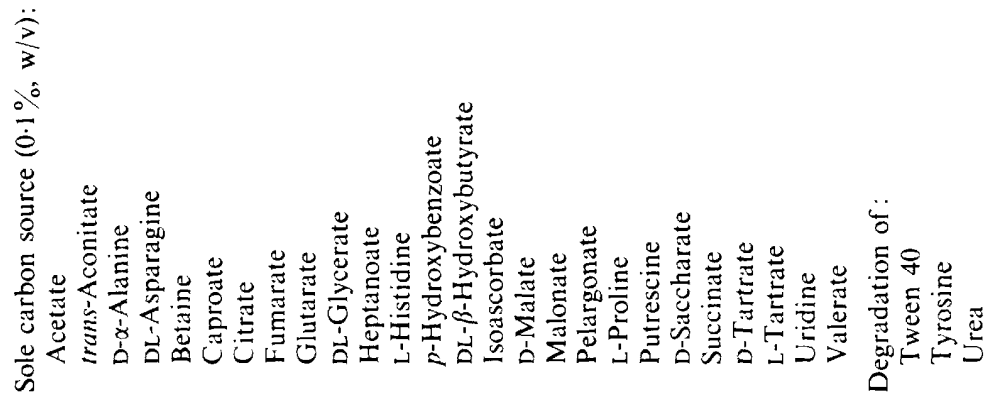




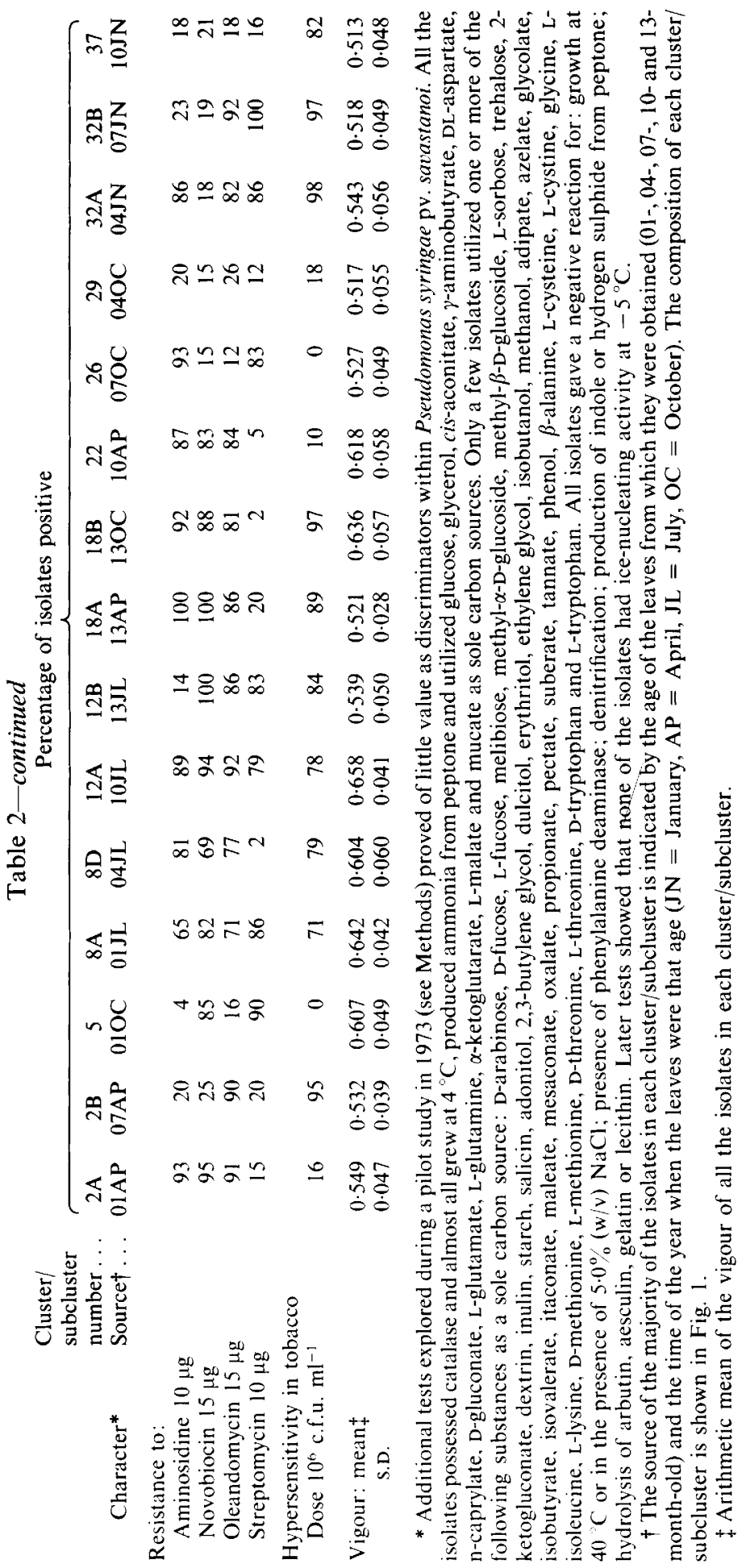


The isolates examined in this study did not differ appreciably in their growth rate. Some of the isolates with a given character in the positive state did differ in the time taken to respond in the pertinent test but the times of test reading were sufficiently delayed to minimize the influence of such differences on the $S$ values. For this reason the results of the $S_{S M} /$ UPGMA analysis gave perhaps a more accurate representation of the potential metabolic diversity of the isolates on the phylloplane. Only 60 characters were determined on each isolate in this study. However, the consistency between the results of different analyses and the invariance of most isolates of $\mathrm{pv}$. savastanoi in 72 additional tests (Table 2) indicated that the observed pattern of relationships between the isolates is soundly based. The relative invariance of pv. savastanoi in the additional tests, most of which are useful discriminators among plant pathogenic fluorescent pseudomonads, also has a taxonomic implication. Since the 60 tests used in this study were selected to give maximum discrimination, the resulting $S$ values were disproportionately low. Therefore caution is needed not to equate the major clusters in Fig. 1 with any formal taxon.

Earlier descriptions of pv. savastanoi agree on the most frequent state of several characters but not of others (Misaghi \& Grogan, 1969; Sands et al., 1970). This makes comparison with the present results difficult. The utilization of lactose, maltose or raffinose, in particular, by some of the epiphytic isolates is in contrast to the data of both Misaghi and Grogan (1969) and Sands et al. (1970). However, acid production was recorded by Dye (1956) from lactose and by Bottalico \& Ercolani (1970) from all three sugars, with at least some of their isolates of pv. savastanoi. Janse (1982) found that 18 isolates of the bacterium causing excrescences on Oleaceae and Nerium oleander were sufficiently distinct from five other members of $P$. syringae to justify subspecies rank for them, as $P$. syringae subsp. savastanoi. However, Janse's differential biochemical tests failed to separate several epiphytic isolates of pv. savastanoi from any of 20 authentic cultures of $P$. syringae pvv. syringae, lachrymans, morsprunorum, pisi and tomato $(\mathrm{G}$. L. Ercolani, unpublished observations). Therefore the name proposed by Dye et al. (1980) for the olive knot pathogen, i.e. P. syringae pv. savastanoi, was retained in this paper.

In principle, the phenetic diversity of phylloplane isolates of pv. savastanoi from different sources may serve as a useful tool in epidemiological studies but this approach requires judgement. Control experiments showed that the characters of the isolates obtained most frequently from leaves of a given age at a given time of the year differed in different groves where several olive cultivars were grown under varied conditions. The significance of individual characters in respect of the ability to colonize the phylloplane under a certain set of conditions has yet to be determined. Therefore, for the present the potential for epiphytic survival of a given phenotype can only be anticipated within the boundaries of the environmental niche where it was found originally.

While this work confirmed that pv. savastanoi undergoes seasonal fluctuation on the phylloplane of the olive (Ercolani, 1970, 1978, 1979), it also poses additional questions about the composition of bacterial populations colonizing the surface of leaves of different ages at different times of the year. Several plasmids have been identified in Italian isolates of pv. savastanoi (Comai et al., 1982) but their role in the regulation of the phenotypic characters considered in this study is not known. Experiments to be described elsewhere showed that sprayinoculation of the leaves with an appropriate mixture of isolates reproduced the alternating prevalence of different populations of pv. savastanoi on the phylloplane as the leaves aged. Then, if the same isolates were inoculated separately, the initial rate of growth of each of them on the phylloplane was invariably greatest under such conditions as could be recorded during the period when that isolate was prevalent on the leaves inoculated with the mixture. The experiments showed also that the phenotypic characters of the isolates remained stable during growth on the phylloplane. This suggests that alternation of heterogeneous populations rather than recurrent modification of a homogeneous population was responsible for the observed phenotypic fluctuation of pv. savastanoi in nature. 


\section{REFERENCES}

Armenise, V. (1950). Cicli di accrescimento e differenziazione delle gemme in piante perenni nel territorio di Bari. VIII. L'accrescimento di Olea europaea L. negli anni 1948-1949. Nuovo giornale botanico italiano 57, 391-417.

Bottalico, A. \& Ercolani, G. L. (1970). Pseudomonas savastanoi (E. F. Smith) Stevens su ligustro giapponese in Puglia. Phytopathologia mediterranea 10, 132135.

Christensen, W. B. (1946). Urea decomposition as a means of differentiating Proteus and paracolon organisms from each other and from Salmonella and Shigella types. Journal of Bacteriology 52, 461-466.

Comai, L., Surico, G. \& Kosuge, T. (1982). Relation of plasmid DNA to indoleacetic acid production in different strains of Pseudomonas syringae pv. savastanoi. Journal of General Microbiology 128, $2157-$ 2163.

Crosse, J. E. \& Garrett, C. M. E. (1963). Studies on the bacteriophagy of Pseudomonas mors-prunorum, Ps. syringae and related organisms. Journal of Applied Bacteriology 26, 159-177.

DYE, D. W. (1956). Oleander knot (Pseudomonas sacastanoi (Erw. F. Smith) Stevens). New Zealand Journal of Science and Technology A 38, 407-411.

Dye, D. W., Bradbury, J. F., Goto, M., Hayward, A. C., LelliotT, R. A. \& Schroth, M. N. (1980). International standards for naming pathovars of phytopathogenic bacteria and a list of pathovar names and pathotype strains. Review of Plant Pathology 59, 153-168.

Ercolani, G. L. (1970). Presenza epifitica di Pseudomonas savastanoi (E. F. Smith) Stevens sull'olivo, in Puglia. Phytopathologia mediterranea 10, 130-132.

Ercolani, G. L. (1978). Pseudomonas savastanoi and other bacteria colonizing the surface of olive leaves in the field. Journal of General Microbiology 109, 245257.

Ercolani, G. L. (1979). Distribuzione di Pseudomonas savastanoi sulle foglie dell'olivo. Phytopathologia mediterranea 18, 85-88.

Fry, J. C., Humphrey, N. C. B. \& Iles, T. C. (1981). Time-series analysis for identifying cyclic components in microbiological data. Journal of Applied Bacteriology 50, 189-224.

Goodfellow, M., Austin, B. \& Dawson, D. (1976). Classification and identification of phylloplane bacteria using numerical taxonomy. In Microbiology of Aerial Plant Surfaces, pp. 275-292. Edited by C. H. Dickinson \& T. F. Preece. London \& New York: Academic Press.

Griffiths, A. L. \& LovitT, R. (1980). Use of numerical profiles for studying bacterial diversity. Microbial Ecology 6, 35-43.

GyllenBerg, H. G. (1963). A general method for deriving determination schemes from random collections of microbial isolates. Annales academiae scientiarum fennicae series $A I V$, biologica 69, 1-23.
Houba, C. \& Remacle, J. (1980). Composition of the saprophytic bacterial communities in freshwater systems contaminated by heavy metals. Microbial Ecology 6, 55-69.

JANSE, J. D. (1982). Pseudomonas syringae subsp. savastanoi (ex Smith) subsp. nov., nom. rev., the bacterium causing excrescences on Oleaceae and Nerium oleander L. International Journal of Systematic Bacteriology 32, 166-169.

KLEMENT, Z. (1963). Rapid detection of the pathogenicity of phytopathogenic pseudomonads. Nature, London 199, 299-300.

Liston, J., Wiebe, W. \& Colwell, R. R. (1963). Quantitative approach to the study of bacterial species. Journal of Bacteriology 85, 1061-1070.

MARTIN, Y. P. \& Bianchi, M. A. (1980). Structure, diversity and catabolic potentialities of aerobic heterotrophic bacterial populations associated with continuous cultures of natural marine phytoplankton. Microbial Ecology 5, 265-279.

Mills, A. L. \& Wassel, R. A. (1980). Aspects of diversity measurement for microbial communities. Applied and Environmental Microbiology 40, 578-586.

Misaghi, I. \& Grogan, R. G. (1969). Nutritional and biochemical comparisons of plant-pathogenic and saprophytic fluorescent pseudomonads. Phytopathology 59, 1436-1450.

Morettini, A. (1972). Olivicoltura, 2nd edn. Rome: Ramo Editoriale degli Agricoltori.

Oliver, J. D. \& Colwell, R. R. (1974). Computer program designed to follow fluctuations in microbial populations and its application to a study of Chesapeake Bay microflora. Applied Microbiology 28, 185-192.

Rosswall, T. \& Kviliner, E. (1978). Principalcomponent and factor analysis for the description of microbial populations. Advances in Microbial Ecology 2, $1-48$.

Sands, D. C., Schroth, M. N. \& Hildebrand, D. C. (1970). Taxonomy of phytopathogenic pseudomonads. Journal of Bacteriology 101, 9-23.

SIERRA, G. (1957). A simple method for the detection of lipolytic activity of micro-organisms and some observations on the influence of the contact between cells and fatty substrates. Antonie van Leeuwenhoek 23, $15-22$.

SNeath, P. H. A. (1968). Vigour and pattern in taxonomy. Journal of General Microbiology 54, 1-11.

SNEATH, P. H. A. \& Johnson, R. (1972). The influence on numerical taxonomic similarities of errors in microbiological tests. Journal of General Microbiology 72, 377--392.

SNeATH, P. H. A. \& Sokal, R. R. (1973). Numerical Taxonomy. San Francisco: Freeman.

WISHART, D. (1969). Fortran II programs for 8 methods of cluster analysis (CLUSTAN 1). Kansas Geological Surcey Computer Contributions no. 38, pp. 1-112. 